Relations industrielles

Industrial Relations

\title{
Bernier, Collette et Anne Filion, À nouveau travail, formations nouvelles
}

\section{Paul-André Lapointe}

Volume 48, numéro 4, 1993

URI : https://id.erudit.org/iderudit/050905ar

DOI : https://doi.org/10.7202/050905ar

Aller au sommaire du numéro

Éditeur(s)

Département des relations industrielles de l'Université Laval

ISSN

0034-379X (imprimé)

1703-8138 (numérique)

Découvrir la revue

Citer ce compte rendu

Lapointe, P.-A. (1993). Compte rendu de [Bernier, Collette et Anne Filion, $\grave{A}$ nouveau travail, formations nouvelles]. Relations industrielles / Industrial Relations, 48(4), 784-788. https://doi.org/10.7202/050905ar

Tous droits réservés (C) Département des relations industrielles de l'Université Laval, 1993
Ce document est protégé par la loi sur le droit d'auteur. L'utilisation des services d'Érudit (y compris la reproduction) est assujettie à sa politique d'utilisation que vous pouvez consulter en ligne.

https://apropos.erudit.org/fr/usagers/politique-dutilisation/ 
La première partie, " théories et méthodes », ne retient malheureusement qu'un seul texte théorique, soit celui de Watson, tiré de Sociology, Work and Industry, dont l'une des principales caractéristiques est d'introduire énormément de confusion entre ce qu'est une théorie et un paradigme, cependant qu'il offre un panorama fort vaste des antécédents et des axes de recherche de la sociologie du travail. Un texte sobre et qui va à l'essentiel présente la méthodologie en sciences sociales.

La partie portant sur l'organisation du travail est bien développée et présente aussi bien des textes classiques dans le domaine (Marglin) que les nouvelles tendances dans le champ de l'organisation du travail, alors que celle portant sur la qualification du travail a le mérite de bien poser le problème de l'ambiguïté du concept de qualification, cependant que des contributions majeures, souvent évoquées il est vrai, demeurent absentes : Braverman, Kern et Shumann, Maurice.

La partie intitulée « L'emploi et l'identité au travail " est très bien documentée et offre une synthèse et des analyses intéressantes de la précarisation de l'emploi et, dans une moindre mesure, de l'ethos du travail, souvent confondu avec le thème de l'identité au travail dont il n'est guère question. Un texte portant sur ce thème, par exemple de Sainsaulieu, aurait donné davantage de consistance à cette section, du moins en regard de l'intitulé de la section.

Si la partie portant sur la division sexuelle du travail constitue une riche et importante contribution de ce manuel, celle orientée vers l'étude du mouvement syndical fait figure de parent pauvre. On n'y retrouve aucun texte et aucune donnée relativement à la situation au Canada ou au Québec. Fort heureusement, le second des deux textes que comporte cette partie présente une très brève synthèse de certains aspects du débat actuel entourant l'avenir du syndicalisme.

L'ouvrage se referme sur une bibliographie thématique riche et pertinente. Soulignons que chacun des textes est précédé d'une présentation brève et intelligente qui pose le problème de fond en plus de le situer dans une perspective plus large.

En somme, un recueil de textes présenté sous forme de manuel qui, pour être imparfait, comme c'est presque toujours le cas dans ce genre d'entreprise toujours difficile à réaliser, n'en demeure pas moins fort utile et bien présenté.

Daniel Mercure

Université Laval

A nouveau travail, formations nouvelles, par Colette BERNIER et Anne FILION, Ottawa, Éditions Agence d'Arc, 1992, 137 p., ISBN 2-89022-283-7

En se proposant, comme elles l'indiquent en sous-titre, de réaliser « un bilansynthèse d'études sur les technologies, les qualifications et la formation dans le tertiaire » les auteures se fixent un objectif ambitieux, voire un peu démesuré, tellement la littérature est abondante, complexe et diversifiée. C'est aussi vouloir produire un ouvrage fort pertinent, à même de remplir un vide important, en produisant une indispensable synthèse susceptible d'éclairer les débats fondamentaux qui ont actuellement 
cours sur l'évolution du travail, non seulement dans le tertiaire, mais également dans les autres secteurs d'activité. Suite à leurs nombreuses études et publications sur le travail et après avoir longtemps œuvré au sein de l'IRAT (Institut de recherche appliquée sur le travail), ce qui est particulièrement vrai pour Colette Bernier, les auteures ont sans doute eu le goût de relever ce défi. Ce dernier était d'autant plus invitant que pour Colette Bernier, c'était une récidive, car elle avait déjà, dans le cadre des études réalisées par I'IRAT, dirigé la publication d'un ouvrage collectif qui se voulait un «bilan-synthèse des connaissances » sur les « nouvelles technologies et [les] caractéristiques du travail " (1983). Ce premier bilan se proposait de faire le point sur le débat au sujet de l'évolution de la qualification du travail. Depuis, comme l'indiquent les auteures, les publications se sont multipliées pendant que les approches et les phénomènes évoluaient considérablement en enrichissant les concepts et en déplaçant les enjeux ainsi que les débats. Un nouveau bilan s'impose donc et c'est ce que les auteures nous proposent dans leur ouvrage.

En raison de son format, plutôt réduit avec à peine 65 pages de texte, et en raison de la volumineuse bibliographie composée de 450 titres qu'il comporte, l'ouvrage se classe plutôt dans la catégorie des instruments de travail et des guides d'introduction que dans celle des savants traités. Sa conception et son organisation en témoignent : une table des matières précédant chacun des chapitres; de nombreux titres et sous-titres, sous chacun desquels un thème est présenté de manière concise; des développements théoriques limités et de nombreuses illustrations à l'aide d'études de cas, dont celles que les auteures ont elles-mêmes réalisées dans les banques, les assurances, le secteur de l'éducation et celui des services publics.

Les auteures ne s'attardent pas au contexte des transformations dans l'environnement du travail qu'elles rappellent brièvement. D'entrée de jeu, elles présentent leur thèse concernant l'émergence " d'un nouveau modèle de la qualification ", ainsi caractérisé par les aspects suivants : une nouvelle conception de la maîtrise exigée lors de l'accomplissement du travail qui s'applique désormais à une fonction élargie et non plus à une tâche étroitement définie; un accroissement de la polyvalence et de la flexibilité dans le cadre de nouvelles formes d'organisation du travail; une utilisation accrue des capacités intellectuelles principalement « axées sur le diagnostic et la résolution de problèmes ", compte tenu de l'abstraction croissante du travail ; un caractère de plus en plus collectif du travail faisant «appel à des aptitudes à la coopération et au travail en groupe »; l'ajout d'une "dimension gestionnaire » à la qualification, pour prendre en compte les objectifs économiques de l'entreprise et une importance accrue accordée à la formation de même que l'établissement de nouveaux liens entre le travail et l'enseignement (p. 10-11).

L'ouvrage est organisé en deux chapitres dont l'un porte sur les nouvelles exigences productives dans le cadre de l'informatisation et sur l'organisation du travail tandis que l'autre est consacré à la formation en entreprise. Vient ensuite une annexe, qu'il aurait été plus juste de nommer un appendice, traitant de la qualification du travail et qui veut, comme l'indique son titre, fournir des « repères méthodologiques pour l'analyse de la qualification ". Le livre se termine par une importante bibliographie divisée selon les chapitres et l'appendice. 
En s'appuyant principalement sur les travaux de Cavestro, Troussier et Zarifian, les auteures retiennent trois changements majeurs concernant le contenu du travail. Il s'agit d'une abstraction croissante du travail, d'un renforcement du caractère collectif du travail et de l'apparition d'une dimension gestionnaire dans la qualification. Cette dernière dimension, qui revêt actuellement une importance stratégique compte tenu des nombreux programmes d'amélioration de la qualité, ne nous apparaît pas suffisamment développée par les auteures.

La section de l'ouvrage portant sur l'organisation du travail aborde un débat tout à fait fondamental aujourd'hui : les changements en cours se traduisent-ils par un remaniement, voire un renforcement, ou un dépassement du taylorisme? Mais, partant de la constatation que l'informatisation « va visiblement de pair » avec la polyvalence, les auteures affirment un peu trop rapidement que « la majorité des études mettent de l'avant la polyvalence des emplois comme un des critères principaux d'une remise en question du taylorisme » (p. 29). Pour appuyer cette affirmation, elles reprennent ensuite les arguments du débat entre Muldur et Verdier au sujet de l'évolution du travail dans le tertiaire, dans le cadre de son informatisation. A notre avis, les auteures insistent trop sur une seule dimension de l'organisation du travail, soit la répartition des tâches en postes de travail qui peut prendre la forme d'une spécialisation extrême (une tâche $=$ un poste) ou d'une polyvalence plus ou moins importante. Dans le cadre de ce débat, elles négligent ainsi d'autres dimensions tout aussi fondamentales, soit la qualification, qu'elles traitent par ailleurs abondamment dans d'autres sections de leur ouvrage, et la répartition des pouvoirs et du contrôle sur le travail. La nouvelle polyvalence se traduitelle par un accroissement ou non de la qualification du travail ? Dans un autre ouvrage, sous la plume d'une des auteurs, C. Bernier, Le travail en mutation (IRAT et SaintMartin, 1990), cette question est très bien posée. Pourquoi ne l'ont-elles pas reprise ici? Cela aurait permis de clarifier grandement les quelques pages qui traitent de la polyvalence et de la spécialisation. Par ailleurs, il aurait fallu s'interroger sur l'évolution du contrôle au sein de l'organisation du travail : avec une autonomie et des responsabilités accrues, les travailleurs et les travailleuses voient-ils leur pouvoir s'accroître sur leur propre travail, et les contrôles hiérarchiques s'affaiblir, ou assistons-nous à l'évolution contraire? Enfin, une mise à contribution d'autres auteurs, comme Coriat, Wood ainsi que Kern et Schumann, aurait bien sûr considérablement enrichi le débat, mais une lecture plus attentive des textes de Muldur et Verdier aurait quand même suffi, il nous semble, pour bien situer tous les enjeux que ce débat soulève.

Dans leur chapitre sur la formation, les auteures présentent trois conceptions de la formation en entreprise qui se sont succédées au cours de la demière décennie : la « formation adaptative ", la « formation qualifiante » et un troisième type de formation, considérée comme « une politique de gestion de la main-d'œuvre ». Alors que le premier type est bien défini et critiqué dans ses insuffisances, les deux autres sont présentés un peu trop rapidement. Corollaire obligé du taylorisme, la « formation adaptative » consiste en "l'apprentissage de procédures simples spécifiques à un poste de travail » (p. 47). Les auteures rappellent les changements au contenu du travail, déjà présentés dans la première partie du chapitre 1, qui rendent ce premier type de formation de moins en moins approprié aux nouvelles caractéristiques du travail. Pour avoir, par contre, une bonne définition de la « formation qualifiante », qui s'inscrit dans un processus de 
requalification et d'enrichissement du travail, il faut se référer à d'autres publications des auteurs, notamment Nouvelles technologies: qualifications et formation (IRAT, cahier 30, 1989). Quant au dernier type de formation, il fait partie d'une politique globale de l'entreprise qui considère les ressources humaines qualifiées et compétentes comme sa principale richesse et la formation, non plus comme une dépense, mais plutôt comme un investissement. Dans ce chapitre, les auteures présentent également des résultats de recherche et de nouvelles approches de la formation, mettant notamment en évidence les pratiques informelles de formation, l'importance de la socialisation et l'effet formateur de l'organisation du travail lorsqu'elle repose notamment sur la polyvalence.

Après quelques précautions d'usage sur la complexité du phénomène, l'appendice sur la qualification s'ouvre sur une « définition opérationnelle » de celle-ci, comme « maîtrise du procès de travail », reprenant ainsi la définition qu'en donnent Rosanvallon et Troussier (1983). Elles ajoutent immédiatement « [qu']une telle définition ne pourrait se passer d'un certain nombre de spécifications concernant la façon d'en analyser les diverses composantes $»$ (p. 72). Pour ce faire, elles opèrent d'abord une excellente critique de l'approche traditionnelle de la qualification exigée par le poste de travail dans le cadre des programmes d'évaluation des tâches. Vient ensuite la présentation de nouvelles approches du phénomène qui constituent autant de dimensions négligées par la première approche : l'apport des ergonomes qui ont bien mis en évidence les compétences réelles mises en cuvre dans l'accomplissement du travail; les qualifications tacites, non officiellement reconnues mais essentielles à la production; la dimension collective de la qualification; la typologie des savoir-faire de Barcet, LeBas et Mercier de même que l'approche de la qualification comme un « fait social », à la conjonction des systèmes d'enseignement, de production et de relations industrielles. Mais, pourquoi ne pas avoir rappelé dans cette présentation la thèse de Zarifian au sujet de la « dimension gestionnaire " de la qualification? Dans cet appendice, qui se proposait de « fournir des repères méthodologiques pour ceux qui aimeraient comprendre la notion de qualification ou encore, entreprendre des recherches sur ce terrain " (p. 71), nous ne retrouvons malheureusement pas des dimensions bien définies, cohérentes entre elles et exhaustives et, encore moins, des indicateurs susceptibles de mesurer le phénomène en question.

L'imposante bibliographie, composée de 450 titres, que nous retrouvons à la fin de l'ouvrage contient nombre de titres fort intéressants et fondamentaux. Elle contient toutefois des lacunes importantes : la production de langue anglaise est peu présente, car elle ne compte à peine qu'une cinquantaine de titres et des ouvrages fondamentaux sont absents. Mentionnons entre autres les Coriat (L'atelier et le robot, 1990), Wood (The Transformation of Work, 1989), Kern et Schumann (La fin de la division du travail?, 1989), De Montmollin (L'intelligence de la tâche, 1984, notamment; un auteur incontournable en ergonomie), Kraut (Technology and the Transformation of White-Collar Work, 1987) et Kling (notamment son article avec Iaconno, « L'informatisation du travail de bureau et l'organisation ", Technologies de l'information et société, 1988). De plus, les auteures n'ont pas suffisamment défini les principes ayant présidé au découpage de leur bibliographie et même à celui des thèses retenues.

Enfin, il nous semble que la spécificité du travail et de la qualification dans le tertiaire aurait dû être posée. Les auteures ont largement utilisé des problématiques déve- 
loppées à la suite de recherche dans le secteur manufacturier, alors que les études de cas présentées concernaient le tertiaire. Pouvons-nous appliquer directement des problématiques du travail manufacturier au secteur tertiaire? Au sein même du tertiaire, ne devrions-nous pas distinguer le travail sur des objets matériels de celui qui s'exerce sur des personnes?

Une dernière remarque : il aurait été important, afin de clarifier l'exposé, de mieux distinguer entre d'une part les approches nouvelles du travail, faisant apparaître des aspects longtemps ignorés du phénomène, et d'autre part les changements récents au niveau du travail, se traduisant par l'émergence de dimensions nouvelles et porteuses d'une certaine transformation du réel.

Néanmoins, l'ouvrage de Colette Bernier et Anne Filion est à lire et à conserver parce qu'il fournit un court résumé de thèses importantes sur l'évolution du travail depuis les années quatre-vingt et qu'il représente une certaine synthèse des débats et des enjeux qui ont actuellement cours dans le monde du travail.

Paul-André LAPOINTE

Université du Québec à Hull

Comprendre le comportement de l'individu au travail : un schéma d'intégration, N. Petersen et R. JACOB, Laval, Québec, Agence d'Arc, 1992, 122 p., ISBN 2-89022-300-0

Des travaux publiés à ce jour sur la description et l'explication des comportements de la personne au travail mettent clairement en évidence la difficulté d'arriver à un consensus sur un modèle intégrateur ouvrant l'accès au labyrinthe des sciences du comportement appliquées à l'étude du travail.

Les auteurs de cet ouvrage d'un peu plus d'une centaine de pages ont effectivement cherché à relever ce défi en présentant un modèle unificateur basé sur l'équation de Kurt Lewin qui fait du comportement une variable dépendante de l'interface personnalité-environnement. Les variables pertinentes à l'explication du comportement sont alors regroupées sous trois grands volets: les caractéristiques de la personne (besoins, valeurs, attitudes, etc.); les caractéristiques de l'organisation selon l'approche sociotechnique et la notion de système-ouvert; enfin, un troisième regroupe des variables de processus symbolisées par des cercles. À l'intérieur de ce dernier volet, le comportement apparaît, non pas en fin de séquence, mais plutôt au centre de sorte qu'on voit mieux le jeu des variables qui le génèrent et les conséquences qui en découlent. Le modèle essaie d'échapper à un raisonnement binaire en faisant appel aux notions de causalité circulaire, de boucles de rétroaction et d'interdépendance entre les variables. En ce faisant, les auteurs maintiennent une sorte d'enchevêtrement de causes et d'effets ajoutant ainsi à la complexité du phénomène à l'étude. L'ouvrage comprend quatre grandes parties dont une première fait un court rappel aux fondements conceptuels et théoriques qui ont servi à concevoir le modèle; les autres traitent en séquence de chacun des volets décrits plus haut. Dans leur introduction, les auteurs qualifient de « représentation simplifiée » le schéma unificateur qu'ils proposent. Il m'apparaît plutôt fort 